\title{
EDITORI E FOGLI PERIODICI NELLA SICILIA FRA SETTE E OTTOCENTO: IL CASO DI MESSINA
}

\author{
Patrizia De Salvo \\ Università degli Studi di Messina
}

RESUMEN: Con este trabajo se quiere poner en evidencia la contribución realizada por las imprentas Mesinesas, en particular por las de los Hermanos del Nobolo, a lo largo camino (iniciado en 1793 con la edición de las primeras Gacetas oficiales) hacia la conquista de la libertad de prensa y la formación de una opinión pública consciente en la Sicilia inglesa. Mediante las numerosas publicaciones periódicas que se realizaron en estos tres decenios, la imprenta del Nobolo ha mantenido la causa constitucional, difundiendo los ideales de independencia y los principios liberales de raíz anglosajona.

Palabras clave: Editores, periódicos, opinión pública, Sicilia, Messina, Italia.

ABSTRACT: Con questo saggio si vuole evidenziare il contributo dato dalle tipografie messinesi, in particolare quelle dei fratelli del Nobolo, al lungo cammino, iniziato nel 1793 con l'edizione delle prime gazzette di stampo legittimista, verso la conquista della libertà di stampa e la formazione di un'opinione pubblica consapevole nella Sicilia inglese.

Attraverso le numerose pubblicazioni periodiche, realizzate nel corso di più di un trentennio, la tipografia del Nobolo ha sostenuto, infatti, la causa costituzionale diffondendo gli ideali d'indipendenza e i principi liberali di matrice anglosassone.

Keywords: Editori, fogli periodici, opinione pubblica, Sicilia, Messina, Italia. 


\section{L'influenza della Rivoluzione francese e la fine delle riforme}

La Rivoluzione francese e la reazione che essa destava in tutta Europa portavano la Sicilia, verso la fine del secolo XVIII, ad avere un governo debole, timoroso e sospettoso, che interrompeva ogni seria riforma'.

Dopo la morte del viceré Caramanico e la congiura di Francesco Paolo Di Blasi, veniva di fatto liquidato qualsiasi residuo di riformismo e la politica borbonica si orientava definitivamente in senso conservatore ${ }^{2}$.

Come presidente del Regno, assumeva il governo dell'Isola l'arcivescovo di Palermo Filippo Lopez y Royo che era descritto, anche nelle cronache coeve, come un uomo «ambizioso, amante e vanitoso di pompe esteriori, pieno di vizi, inadatto a tanta carica, inquisitore sospettoso e tirannico ${ }^{3}$, circondato da spie e da delatori. Egli dava inizio alle persecuzioni contro quelli che erano sospetti di coltivare ideali democratici: gli arresti, i processi e le condanne per giacobinismo, ordinati dalla Giunta di Stato, si moltiplicavano di giorno in giorno, suscitando il terrore in tutte le classi sociali:

«nei Baroni, che detestavano quello spaventevole potere assoluto; nella classe media, che anelava di acquistare una moderata libertà; e nel popolo intollerante di servitù e bramoso di scuotere il giogo che l'opprimeva da secoli» ${ }^{4}$.

Si era instaurato un regime fondato sulla paura:

«Si spiavano le opinioni dei cittadini, s'intercettavano le comunicazioni fra I'Isola ed il Continente; s'imprigionava; si esiliava ad arbitrio; era reato perfino la lettura dei giornali con un certo diletto; si faceva guerra ai calzoni lunghi, alla barba ed alle code accorciate, indizî certi di giacobinismo» ${ }^{5}$.

1. Sulle politiche riformatrici del Settecento siciliano, si veda Brancato, F., I/ Caracciolo e il suo tentativo di riforme in Sicilia. Palermo, 1946; Coco, A., "La Sicilia di Caramanico fra «ardita novità» e crisi di fine secolo". De Lorenzo, R., L'organizzazione dello Stato al tramonto dell'Antico Regime. Napoli, 1990; Romano, S. F., Intellettuali riformatori e popolo nel Settecento siciliano. Pisa, 1983; più in generale Giarrizzo, G. (ed.), La Sicilia dal Vespro all'Unità. Torino, 1974.

2. A proposito della vicenda culturale siciliana del secolo XVIII si rinvia a Giarrizzo, G., "Ricerche sul Settecento italiano. Appunti per la storia culturale della Sicilia settecentesca". Rivista Storica Italiana LXXIX (1967), pp. 573-627; per una puntuale analisi degli studi sulle istituzioni politiche e giuridiche nella Sicilia settecentesca, cfr. Novarese, D., "Pragmaticas omnes pristinae integrati restituimus. Francesco Paolo Di Blasi e la raccolta di Prammatiche del Regno di Sicilia (1791-1793)". Atti della Accademia Peloritana dei Pericolanti Classe di Scienze Giuridiche Economiche e Politiche LXXIII (2004) p. 193-231.

3. Cfr. Bianco, G., La Sicilia durante l'occupazione inglese (1806-1815). Palermo, 1902, p. 10.

4. Bianco, La Sicilia durante l'occupazione inglese, p. 10.

5. Bianco, La Sicilia durante l'occupazione inglese, p. 11. 
In quella situazione di persecuzioni e controlli vedevano la luce i primi veri giornali che venivano letti non solo da studiosi e letterati, ma anche, come evidenzia Mario Infelise, da un pubblico di "dilettanti", spesso colto, che aumentava nel corso degli anni ${ }^{6}$.

\section{Prime espressioni di "libertà" di stampa a Messina}

Nonostante tale clima, uno storico locale ricordava che «Fu nel 1793, che i messinesi cominciarono ad apprezzare, una o due volte la settimana, la libertà di stampa» ${ }^{7}$.

La stampa peloritana, in effetti, appariva in quegli anni più attenta, rispetto a quanto accadeva nel resto dell'Isola, alle notizie provenienti dalla Francia ${ }^{8}$.

Causa di ciò poteva essere probabilmente I'interesse e la curiosità stimolati dagli avvenimenti europei che avevano visto la migrazione a Messina di numerosi stranieri ${ }^{9}$, una vera ricchezza per la città che aveva fondato le proprie fortune sui traffici, sui commerci ma anche sulla particolare natura borghese dei suoi ceti ${ }^{10}$.

Era proprio nella città dello Stretto che, sulla scia degli avvenimenti politicomilitari scaturiti dal 1799 e dei nuovi assetti statuiti dalla pace di Firenze, s'impiantavano, più numerosi che nelle altre città dell'Isola, comunità di tedeschi, svizzeri e austriaci, che andavano ad aggiungersi alla borghesia imprenditoriale e commerciale autoctona e a quegli stranieri che si erano insediati nella città peloritana, già dal 1784, attratti dal privilegio del porto franco.

6. Infelise, M., Prima dei giornali: Alle origini della pubblica informazione. Roma-Bari, 2005.

7. Arenaprimo, G., La stampa periodica in Messina dal 1675 al 1860. Messina, 1893, p. 37.

8. Quando nel 1798 I'esercito di Napoleone invadeva Napoli, Ferdinando fuggiva a Palermo con tutta la corte. II suo arrivo era accolto con gioia perché sembrava indicare finalmente I'indipendenza da Napoli e un proficuo ritorno alla vita di corte. L'Isola dava a Ferdinando l'appoggio assoluto e incondizionato contro le truppe francesi e allo stesso tempo beneficiava molto della presenza della corte e di numerosi stranieri.

9. In quel periodo, infatti, la presenza inglese nell'isola diminuiva a favore di una più massiccia presenza a Malta, che era sotto il diretto controllo inglese proprio da quell'anno; a questo proposito si veda, D'Angelo, M., Mercanti inglesi in Sicilia (1806-1815), Rapporti commerciali tra Sicilia e Gran Bretagna nel periodo del blocco continentale. Milano, 1988.

10. Sulla connotazione di Messina come città borghese, si veda: Romano, A., "Élites culturali, élites politiche e cultura giuridica a Messina fra Cinque e Seicento". Romano, A., Istituzioni politiche e giuridiche e strutture del potere politico ed economico nelle città dell'Europa mediterranea medievale e moderna, "la Sicilia". Messina, 1992, pp. 115-147; Novarese, D., luris doctores e giudici a Messina fra Cinque e Seicento: nota per una storia delle élites culturali e politiche siciliane. Romano, Istituzioni politiche, pp. 65-113. Per quanto riguarda la Messina ottocentesca, si veda Chiara, L., Messina nell'Ottocento. Famiglie, patrimoni, attività. Messina, 2002, e bibliografia ivi citata. Da ultimo, Bottari, S. - Chiara, L., La lunga rincorsa. Messina dalla rivolta antispagnola al terremoto del 1908. Manduria-Bari-Roma, 2009. 
Verosimilmente la stampa periodica cittadina aveva, dunque, come utenti gli appartenenti a quella ricca borghesia che, lontana dagli intrighi della corte palermitana, era interessata ai movimenti di capitale e di manodopera e non poteva non tener conto di quanto avveniva nel continente.

La tipografia dei Nobolo fondava proprio in quegli anni la sua fortuna attraverso una serie di pubblicazioni, attente alle vicende rivoluzionarie francesi e alle prime imprese napoleoniche, che soddisfacevano l'esigenza insita nella natura borghese della città.

I due fratelli Giovanni e Giuseppe avrebbero stampato ininterrottamente per un lungo periodo, segnando, peraltro, il cammino della formazione dell'opinione pubblica nella città dello Stretto, passando dalla pubblicazione di gazzette legittimiste all'edizione di giornali costituzionali"11.

Il primo foglio, pubblicato per i tipi dei fratelli del Nobolo, era il Compendio delle Notizie più recenti, edito a Messina il 16 marzo del 1793 a cura di don Antonino Pantò. Dopo I'edizione dei primi fascicoli, I'abate Pantò trasferiva I'incarico della stampa di quel foglio alla tipografia di Giuseppe Di Stefano. Per tutta risposta, a distanza di qualche mese, i del Nobolo, coadiuvati da Candeloro Melardi ${ }^{12}$, cominciavano le pubblicazioni di un altro periodico settimanale, il Corriero di Messina, che sembra avere avuto maggiore fortuna del primo.

La fonte ufficiale, per entrambi i giornali, era la polizia che, interessata a screditare gli ideali giacobini e la Rivoluzione francese, forniva il più delle volte notizie false o poco attendibili. I lettori, lontani dai principali centri d'informazione, appagavano in tal modo la loro curiosità e marginale diventava la veridicità delle notizie.

Ciò nonostante si può affermare che simili pubblicazioni periodiche rappresentavano per Messina un primo passo verso la via del progresso e dell'emancipazione civile.

11. A proposito dello sviluppo dell'arte della stampa a Messina, cfr. Molonia, G. (ed.), Cinque secoli di stampa a Messina. Messina, 1986, pp. 231-242. Su i giornali costituzionali editi dalla tipografia del Nobolo, si veda De Salvo, P., "Civil and political liberties: the debate on early nineteenth century sicilian press". Parliaments: The law, the practice and the representations. From the Middle Ages to the Present Day. Coimbra 2010, pp. 455-460. La tradizione tipografica dei Nobolo sarebbe stata continuata dal figlio di Giuseppe, Michelangelo, attivo oltre l'unificazione del Regno d'Italia.

12. Il Melardi veniva descritto come un «litteratonzolo stipendiato da Maria Carolina, [che] gonfiava col cannello le bolle di sapone: era lui che impiastricciava proclami e decreti, che magnificava le imprese e le gesta del vicario generale, e che non stancavasi dal ripetere che "tutta Europa erasi, come un solo uomo, levata in armi al soccorso dei sovrani fuggitivi", e che gli eserciti francesi e dei patriotti erano stati tagliati a pezzi in ogni parte d'Italia [...]. Con siffatte arti, con tante bugie, scoraggiando i carbonari ed i liberali del tempo, guidavasi la pubblica opinione a pro della controrivolta», cfr. Arenaprimo, La stampa periodica a Messina, pp. 42-43. 
L'anno successivo, nel mese di aprile, le due testate si fondevano in un nuovo foglio dal titolo Compendio delle Notizie più recenti del Corriero di Messina.

La logica di tale unione si leggeva nel fascicolo numero 15, del 12 aprile:

«[...] ci siamo indotti sempre a maggior serviggio di questo rispettabilissimo Publico, e dei nostri rispettivi Sig. Associati a secare le contese [e] unirci, ed a migliorare i nostri fogli riducendoli ad uno solo per settimana, il quale partecipando del titolo dell'uno e dell'altro si chiamasse per l'avvenire II Compendio delle notizie più recenti del Corriero di Messina. E ciò all'oggetto di poter Noi avere un campo più comodo a poter dar piacere nel rapporto dell'avvenimenti dell'a questa ora novella entrata Campagna delle Potenze Alleate, contro la Contumace Republica di Francia ${ }^{13}$.

Anche questa pubblicazione, come si evince dal redazionale, aveva come scopo la propaganda realista. A Messina, infatti, nonostante i numerosi tentativi, anche a mezzo stampa, di screditare le nuove dottrine rivoluzionarie e l'attivissima sorveglianza della polizia, erano presenti focolai giacobini, come ricordava Gaetano Oliva:

«Non mancavano, infatti, fra noi, benché sulle prime in iscarsissimo numero, coloro che aspiravano ad una riforma della vecchia società; ed il governo, che ne vedeva i prodromi, si cooperava a tutt'uomo a scongiurare la futura tempesta. Si mise ogni opra per screditare la rivoluzione francese, ed oltre che le settimanali gazzette, altre pubblicazioni si fecero a Messina, tutte intente a siffatto scopo, non essendosi credute neppur sufficienti le molte e continue prediche che si facevano dai sacerdoti in tutte le chiese della città, i quali allora per conto proprio, ed eziandio per conto del governo aveano mutata la cattedra del vangelo in politica tribuna» ${ }^{14}$.

Il sodalizio che aveva visto nascere il Compendio durava solo un anno: nel 1795, infatti, riprendevano le pubblicazioni del Corriero di Messina che, sempre per i tipi di del Nobolo, sarebbe stato stampato fino al 1798.

Un nuovo foglio settimanale, edito dal tipografo Giuseppe Di Stefano, dal titolo Nuove di diverse Corti e Paesi, vedeva la luce nel corso del 1796: anch'esso attingeva le sue notizie dai rapporti di polizia e sosteneva la causa monarchica. Quando la redazione si trovò nella condizione di poter sconfessare le notizie false e rivelare, seppur in parte, la verità sullo stato politico e militare della Penisola italiana, sulle molte sconfitte sofferte dagli alleati e sulle vittorie dei repubblicani, dati sempre per perdenti dalle fonti ufficiali, la sua vita divenne difficile e ben presto non fu più pubblicato. La soppressione di quella

13. Compendio delle notizie più recenti del Corriero di Messina, sabato 12 aprile 1794, n.15, p. 1.

14. Cfr. Gallo, C. D.-Oliva, G., Gli Annali della città di Messina. Messina, V, 1893, p. 189. 
gazzetta, che al suo apparire aveva ottenuto un certo apprezzamento, passava, tuttavia, quasi inosservata.

Con la pace di Firenze del 1801, si sanzionava una nuova sconfitta dei Borbone. I nuovi accordi con la Francia napoleonica prevedevano, oltre alla presenza di truppe francesi nel Regno di Napoli, la riapertura di tutti i porti del regno alle navi francesi e la chiusura degli stessi a quelle inglesi. Nonostante il trattato di pace, e una maggiore tranquillità nei rapporti tra Napoli e la Francia, la parte culturalmente più attrezzata della cittadinanza messinese viveva in continua apprensione per le clamorose vittorie riportate da Napoleone Bonaparte e per le modifiche istituzionali dei paesi assoggettati.

La curiosità verso gli avvenimenti politici, i timori e le speranze che le notizie suscitavano, portava alla considerazione che il mondo delle gazzette cittadine fosse troppo limitato: il solo Corriero di Messina non era sufficiente a soddisfare il desiderio d'informazione del pubblico lettore che, in città, aveva i suoi punti di ritrovo presso la libreria di Luigi Caccia Spadaro e il caffè dei nobili che situato nella piazza del Duomo

«sin dal principio del secolo fu anche uno dei convegni della classe pensante del paese, e di tutti coloro che avevano tempo da perdere e chiacchiere da contare. Avendo riguardo alle condizioni di quei tempi, erano le farmacie ed i pochi caffè il solo mezzo concesso per lo scambio delle idee e dove potea maturarsi la pubblica opinione ${ }^{15}$.

A colmare un tale vuoto si aggiungeva la pubblicazione di un foglio P. S. $O$ notizie epilogate e, con cadenza mensile, del Giornale Istorico, Politico e Militare che riassumeva le vicende e gli episodi più importanti della guerra in corso in Europa.

Queste nuove pubblicazioni non corrispondevano, tuttavia, alle esigenze della popolazione messinese desiderosa di un giornale di più grande formato, redatto in maniera più completa e puntuale, che costasse meno e quindi alla portata di un maggior numero di lettori e che fosse, soprattutto, più imparziale nella scelta delle notizie. L'Osservatore Peloritano, pubblicato dall'infaticabile Giovanni del Nobolo tra il 1797 e il 1804, rispondeva a questi requisiti. Infatti, accanto ad una copiosa raccolta di notizie provenienti dall'estero e dai territori italiani, vi si trovavano, di volta in volta, articoli di genere letterario e politico,

15. Questa libreria era il ritrovo di quanti «per virtù e per ingegno onorassero allora il paese. Frequentavano assai spesso il locale Andrea Gallo, Emanuele Taddei delle Scuole Pie, il barone Placido Arena-Primo, il sacerdote Alberto Corrao, professore di diritto canonico nell'Accademia Carolina degli studî, Ottavio Saccano, il marchese Agostino Cardillo, i fratelli Arrosto, monsignor Gaetano Grano (1754-1828), ed il sacerdote Antonio Jacii. Talora vi si leggevano dissertazioni, e quasi sempre poi eran vive le dispute». Arenaprimo, La stampa periodica a Messina, nt. 2, pp. 44-45. 
annunzi legali, rassegne commerciali e spesso vi si poteva leggere un cenno alla cronaca cittadina. Aveva una notevole diffusione e fortuna tanto da essere ricordato per essere stato «la delizia di tutte le persone culte della città ${ }^{16}$.

Attraverso le pagine dell' Osservatore Peloritano la redazione dimostrava il suo totale asservimento alla corona «sperticando lodi all'atteggiamento della Gran Bretagna, alle virtù di re Ferdinando, ed all'angelica figlia di Maria Teresa», e assicurando quotidianamente che «vescovi e gentiluomini si offrivano per guerrieri a sostegno della Corona, e che una guerra più santa di questa non si era combattuta mai al tempo delle Crociate, sotto Goffredo e Riccardo Cuor di leone ${ }^{17}$.

Nel 1803 era edito, con l'obbiettivo di divulgare le scoperte scientifiche, le novità letterarie e riportare le rassegne bibliografiche, il Foglio di Letteratura, Scienze, Arti e Commercio. L'importanza di questo giornale era accentuata dal circuito di distribuzione in cui era inserito. Era venduto, infatti, non solo a Messina, presso le librerie di Nicolò Di Stefano e di Luigi Caccia Spadaro, ma anche a Palermo (presso il negoziante di libri Rosario Abbate), a Catania (presso il libraio Stefano Caccia Spadaro) e a Siracusa (dal cavaliere fra Luigi Bongiovanni).

L'anno successivo Giovanni del Nobolo dava alle stampe il Corriere Nazionale che mutava quasi subito il titolo in Corriere Messinese. Si trattava di un foglio di notizie varie. Sembrerebbe il primo tra i giornali cittadini a non ricevere direttamente le informazioni dalla polizia o dal governatore politico e militare della città ${ }^{18}$.

La ripresa delle ostilità tra la Francia e i Borbone nel 1806, I'occupazione del Regno di Napoli e la nuova fuga di Ferdinando IV in Sicilia permettevano più stretti rapporti tra Messina e I'Inghilterra. I nuovi movimenti di truppe nel sud della Penisola e l'arrivo degli inglesi in città, portavano l'editore Nobolo a stampare, dal 5 luglio 1806 sino ai primi di febbraio del 1807, un nuovo foglio dal titolo Notizie Officiali che riportava le vicende della spedizione dell'armata inglese in Calabria ${ }^{19}$.

16. Venuto alla luce nel 1797, era pubblicato una volta la settimana fino al 20 aprile 1804, data in cui veniva edito da un altro tipografo e da una nuova redazione. Sarebbe durato per circa mezzo secolo. La raccolta si trova presso la Biblioteca del Museo di Messina. Cfr. GalloOliva, Gli Annali della città di Messina, p. 199.

17. Cfr. Arenaprimo, La stampa periodica a Messina, pp. 41-42.

18. Per quel che ho potuto vedere, dalla raccolta conservata nella Biblioteca del Museo Regionale di Messina, la redazione non seguiva nessuna indicazione politica prestabilita, si limitava a riportare notizie riferendole ai paesi di provenienza, alcune volte, nella quarta pagina, alla fine del fascicolo, si trovavano avvisi e annunci pubblicitari.

19. Le prime notizie su questo foglio si leggono in Arenaprimo, La stampa periodica a Messina, p. 46. Durante una fortunata ricerca presso la Biblioteca del Museo Regionale di Messina ho rinvenuto la maggior parte di questi fogli. Ė da questa pubblicazione che Giovanni del Nobolo comincia a firmarsi "Impressore Britannico". 
Oltre ai giornali già presenti in quei primi anni del secolo XIX, vedeva la luce nel 1808, dapprima come quindicinale e poi come settimanale, la Conversazione Istruttiva, ossia Foglio Interessante. Era un giornale "didattico" utile per divulgare, in maniera semplice, alcune nozioni elementari, impostandole a mo' di dialogo con una dama, un cavaliere, un avvocato o un filosofo. Spesso la redazione del giornale lasciava trasparire i propri orientamenti politici, soprattutto quando dedicava sonetti alla bellezza delle donne inglesi $i^{20}$. Nello stesso anno era edita sempre, per gli stessi tipi, la Gazzetta Britannica.

A queste opere a stampa, nel 1811, se ne aggiungevano altre due: il Giornale di Scienze, Lettere e Arti ${ }^{21}$, foglio ufficiale della Reale Accademia Peloritana, e il Logogrifo o Monitore segreto, che aveva come funzione principale quella di denigrare la persona e le imprese di Napoleone: a tal fine sul frontespizio portava il motto Son empire est detruit si l'homme est reconnu.

È possibile catalogare queste diverse pubblicazioni secondo dei criteri ben precisi: se alcune di queste avevano per lo più il carattere di riviste culturali, come il ricordato foglio ufficiale dell'Accademia dei Pericolanti o la Conversazione Istruttiva, altre, la maggior parte, erano vere e proprie pubblicazioni d'informazione. Esse facevano parte della folta schiera di ben note gazzette che, con titoli diversi, venivano edite in ogni parte della Penisola italiana e d'Europa, e che univano in un'unica raccolta: atti ufficiali, discorsi, proclami privati, poesie e componimenti letterari d'occasione.

Da tali opere si distingueva la Gazzetta Britannica giornale squisitamente politico: nei sette anni di pubblicazione avrebbe trattato, infatti, argomenti per lo più relativi alla vita politico-istituzionale dei regni dei Borbone e dell'Impero britannico ${ }^{22}$.

20. si veda Conversazione Istruttiva, n. 11, sabato 15 ottobre 1808.

21. Il sodalizio messinese, in quegli anni, viveva un periodo di fertile attività: numerosi erano i contatti e gli scambi che esso intratteneva con altre accademie scientifiche e letterarie d'Europa. Sin dai primi anni dell'Ottocento, era stato desiderio comune del consesso peloritano di avere un proprio settimanale, ma l'interruzione del servizio postale con il continente a causa delle vicende politiche e belliche, portava i soci ad abbandonare l'iniziativa. Con lo stanziamento delle truppe inglesi, e la conseguente riapertura del servizio di posta con alcune regioni della Penisola e con I'Inghilterra, si realizzavano quelle circostanze che portavano alla stampa, per i tipi dei soci Letterio Fiumara e Giuseppe Nobolo, del nuovo periodico. Accanto alle dissertazioni letterarie e filosofiche spesso trovavano spazio, tra le pagine del settimanale, articoli carichi di attacchi e critiche contro il giacobinismo.

22. A proposito della Gazzetta Britannica, periodico bisettimanale edito a Messina dal 1808 al 1814 , mi sia consentito ricordare che, nell'ambito di un progetto di ricerca d'Ateneo, da me coordinato, dedicato all'analisi del rapporto tra potere politico e opinione pubblica nella Sicilia inglese (1806-1814), è prevista la ristampa anastatica della Gazzetta preceduta da un ampio studio introduttivo. 


\section{La presenza inglese in Sicilia e la libertà di stampa}

Dopo la proclamazione dell'Impero, la progressiva estensione a tutta la Penisola italiana degli istituti e degli ordinamenti napoleonici riguardava anche il regime di stretta sorveglianza cui era sottoposta la stampa. Attraverso successivi inasprimenti della censura, si giungeva al diretto controllo del governo sui fogli parigini e francesi in genere. Questo, infatti, oltre a nominare un capo redattore e un censore, obbligava le redazioni di provincia, con una circolare del 6 novembre 1807, a riportare solo articoli politici ricopiati dal Moniteur, il giornale ufficiale.

Negli anni successivi, con nuove leggi si limitavano le testate giornalistiche a una sola per Dipartimento e a quattro per Parigi ${ }^{23}$.

Anche i pochi giornali editi a Napoli, in quegli anni, e primo fra tutti il Monitore Napolitano, erano, dunque, diretta emanazione delle autorità ${ }^{24}$. II primo numero del Monitore, pubblicato sabato 1 marzo 1806, si rivolgeva ai lettori con queste parole:

La Gazzetta Napolitana è soppressa. La saviezza, la dignità e la franchezza di un Governo ragionevole, generoso, e giusto non dovea soffrire, che così vilmente si nascondesse, o mascherasse la verità agli occhi della nazione per servire ad una debole, sospettosa, e inconseguente politica. Se giudicar si dovesse del carattere di una nazione, che non ebbe mai altra parte nelle operazioni del suo Governo, che quella di soffrire in silenzio, e di tremare, se io dico, giudicar si dovesse dal racconto dei fatti o alterati, o inventati a bella posta dalla penna venale di un Giornalista, avrebbero per avventura gli stranieri ragion di credere, che il più bel paese di Europa sia da barbari, o da selvaggi abitato. Ma un popolo conosciuto per lo genio, che I'ha distinto, e per li talenti, che l'hanno illustrato, non riceverà presso i saggi estimatori delle cose alcun torto dalla imbecillità di un Gazzettiere. Avrebbe meritata forse qualche indulgenza, se si fosse contentato di tacere sulle strepitose vittorie riportate dalle armate Francesi in Alemagna. S'intendeva bene, che egli mantener doveva nell'illusione un popolo, che la Corte spinger voleva alla guerra. Ma come mai potrebbe giustificarsi oggigiorno? Passar sotto silenzio un avvenimento sì fausto, sì glorioso, come l'ingresso è stato delle Armi Francesi in

23. Cfr. Capra, C., Castronovo, V., Ricuperati, G., La stampa italiana dal ' 500 all' 800 . RomaBari, 1986, pp. 487-532. Scotti Douglas, V., "Los periódicos italianos, la propaganda napoleónica, y la Guerra de la Independencia". Trienio Ilustración y Liberalismo 52 (2008), pp. 97-139.

24. I provvedimenti di legge, presi dal governo francese a Napoli e volti a censurare la stampa erano: un parere del Consiglio di Stato a favore della continuazione dei giornali di intendenza, e il decreto del 30 novembre 1810 che estendeva al Regno di Napoli le norme francesi sulla riduzione del numero delle testate. In virtù di tale provvedimento, e sulla scorta di un nuovo decreto si ordinava la fusione dei due giornali politici esistenti, nel Monitore delle Due Sicilie (cfr. Zazo, A., II giornalismo a Napoli nella prima metà del secolo XIX. Napoli, 1985, p. 339). 
Napoli, le circostanze che I'hanno accompagnato, I'arrivo di S. A. I. il Principe Napoleone Giuseppe, le prime sue operazioni, è un tratto di malignità il più ridicolo, il più indecente, il più odioso ${ }^{25}$.

Appare chiaro come, in tal modo, fosse gradualmente eliminata ogni forma, seppur minima, di dissenso e di critica delle questioni politiche.

Dall'impero napoleonico rimanevano fuori, com'è noto, grazie alla protezione della flotta inglese, la Sicilia e la Sardegna, rifugio, la prima, della monarchia borbonica e, la seconda, della corte sabauda.

Il governo di Londra, per parte sua, utilizzando le stesse armi del nemico, faceva appello al sentimento nazionale e al desiderio di libertà degli italiani, per istigare, attraverso la carta stampata, I'opposizione al regime napoleonico. Uno dei maggiori centri della diffusione di notizie antifrancesi era l'isola di Malta che, grazie alla sua posizione al centro del Mediterraneo, era particolarmente adeguata a fungere da base per il traffico illecito di merci e idee ${ }^{26}$. Deus ex machina di questa propaganda era Vittorio Barzoni ${ }^{27}$, uno dei primi e più appassionati oppositori italiani di Napoleone. Espulso dall'Austria, aveva trovato a Malta asilo politico. Subito gli inglesi mettevano a frutto le sue capacità di pubblicista per dar vita a numerose esperienze editoriali di matrice antinapoleonica, tra cui il Giornale di Malta che, scritto proprio dal Barzoni in collaborazione con Giuseppe Casolani, e pubblicato a partire dal 7 gennaio 1812, riportava sulla testata lo stemma inglese che aveva contraddistinto, sin dal suo nascere, il 2 marzo del 1808, anche la Gazzetta Britannica di Messina.

\section{La Gazzetta Britannica di Messina}

Le circostanze che portavano alla pubblicazione di un vero e proprio giornale politico, con ampie rubriche dedicate alle notizie italiane ed estere, e un ben preciso orientamento critico, erano date dal determinarsi di una situazione quanto mai delicata nella città dello Stretto.

25. II Monitore Napolitano, n. 1, 1 marzo 1806, p. 1.

26. A tale proposito si rinvia a De Salvo, P., "Opinione pubblica, libertà di stampa e circolazione delle idee: I'influenza inglese nel mediterraneo (1790-1817)", contributo al Workshop: Public opinion, politics and press in Europe (1789-1914), Santander, 25-27th November 2010, ora in corso di stampa.

27. Sul Barzoni si veda Nuzzo, G., "Barzoni Vittorio". Dizionario biografico degli italiani, 7, Roma, 1965, pp. 41-44; Renucci, G., "Profilo di Vittorio Barzoni". Rivista italiana di studi napoleonici IX (1970), n. 2, pp. 136-150, e bibliografia ivi citata; da ultimo, cfr. Portelli, S., "Una penna italiana al servizio di Sua Maestà: la propaganda filo britannica di Vittorio Barzoni nel Mediterraneo, 1804-1814". Rivista di studi napoleonici XXXVII (2004), n. 1, pp. 33-49. 
Fra la borghesia cittadina, stanca dei soprusi della monarchia borbonica, $\mathrm{s}^{\prime}$ insinuavano atteggiamenti ideologici francofili e tra le due sponde dello Stretto si riscontrava un certo "contrabbando" d'idee che preoccupava il generale Stuart, comandante in capo dell'armata britannica in Sicilia. Questo spiega il compito della nuova gazzetta che, attraverso una vera e propria "guerra psicologica"28, doveva replicare al fermento sotterraneo cui gli agenti francesi davano vita, sull'altra sponda, attraverso l'introduzione di fogli volanti, avvisi e proclami, e bloccare così la diffusione di correnti rivoluzionarie nella società messinese.

Sin dal manifesto riprodotto sul primo fascicolo, volto a spiegare le ragioni della nuova pubblicazione, si possono individuare le origini di quello che era I'humus sul quale si sarebbe sviluppato il giornale: la critica a «un inopportuno silenzio» e alla «totale soppressione dei pubblici fogli» era la conseguenza del riconoscimento dell'importanza attribuita alla pubblica opinione dalla tollerante tradizione inglese. E se la prima annata si era profusa in una campagna denigratoria contro Napoleone Bonaparte, a tutto vantaggio dei legittimi sovrani borbonici, è possibile individuare i segni del mutamento già dalla seconda annata, densa di notizie dedicate soprattutto alla guerriglia sostenuta dall'armata britannica contro le forze napoletane e murattiane presenti sulla costa calabrese, che metteva in luce il coraggio della flotta anglo-sicula in contrapposizione alla codardia dei napoleonici.

Certamente I'ammirazione che la Gran Bretagna riscuoteva nell'opinione pubblica siciliana nei primi anni del secolo XIX è da ricercarsi in quella diffusione che la cultura inglese aveva avuto nell'Isola e in generale in tutta I'area del Mediterraneo dalla seconda metà del Settecento ${ }^{29}$.

28. È Giorgio Spini che parla di psycological warfare in "A proposito di «circolazione delle idee» nel Risorgimento: La "Gazzetta Britannica» di Messina". Spini, G., Incontri europei e americani col Risorgimento. Firenze 1988, pp. 15-36.

29. Sono testimonianza di questo interesse verso la cultura inglese i rapporti diretti tenuti dal filosofo di Modica Campailla con il Berkeley; lo storico Cubani con I'Hoyvon, il Wodvard, lo Scherard, il Pitton; I'economista Balsamo con Young. Come pure, erano componenti dell'Accademia di Londra il Principe di Biscari, il Caruso, il Paternò di San Giuliano. D'altra parte, non pochi erano i viaggiatori inglesi che visitavano I'Isola in quel torno d'anni; cfr. De Mattei, R., II pensiero politico siciliano fra il sette e l'ottocento. Catania, 1927, p. 48. Il Brydone, nel suo viaggio in Sicilia nel 1770, rimaneva favorevolmente sorpreso dalla facilità con cui gli appartenenti all'alta società conversavano con lui in inglese, al punto da scrivere nel suo diario di viaggio: «Noi ci eravamo stupiti arrivando a Palermo di trovarvi dei gentiluomini che ci parlavano inglese, ma noi lo fummo ben più quando essi ci provarono di conoscere perfettamente parecchi dei nostri migliori Poeti e dei nostri Filosofi. Noi abbiamo trovato in originale, in parecchie biblioteche, le migliori edizioni di Milton, Shakespeare, Dryden, Pope, Bacon, Bolingbroke. La nostra lingua è talmente divenuta di moda che la si considera come parte essenziale di buona educazione. II Viceré e il Marchese Fogliani, uomo di gran merito, hanno fatto uno studio particolare di alcuni nostri autori, ed essi incoraggiano i progressi che queste conoscenze fanno nel regno»; il brano si legge in De Mattei, Il pensiero politico, p. 49. 
Tale apprezzamento era anche testimone dell'interesse con cui i "dotti siciliani" guardavano alle istituzioni della Gran Bretagna che rendevano gli inglesi «uomini forti, magnanimi, indipendenti e dominanti in tutte le quattro parti del globo $»^{30}$.

Come era avvenuto a Malta ${ }^{31}$, infatti, anche in Sicilia e in particolare a Messina iniziava, sotto la protezione dell'Armata britannica di stanza in città, un'attenta campagna antigiacobina e antinapoleonica proprio attraverso le pagine della Gazzetta Britannica e, non era un caso, che la redazione, nel licenziare il primo fascicolo, sottolineasse, ispirandosi al modello del liberalismo inglese, I'importanza della libertà di stampa con il seguente programma:

«Mentre dei grandi avvenimenti hanno sorpreso tutto il mondo, ed hanno dato materia di arricchir di volumi la storia dei nostri tempi, un inopportuno silenzio è accaduto, e la totale soppressione dei pubblici fogli. Da ciò n'è seguito che alcuni, o capricciosi o torbidi, hanno spacciato delle novelle o alterate, o intieramente false, sostenute sovente da alcuni fogli francesi o italiani, che sono ad arte foggiati per sostenere, o legittimare gl'interessi degli usurpatori Corsiani. Motivi son questi che han fatto dare alla luce la presente Gazzetta, la quale non rapporterà che fatti, e che nel tempo istesso farà conoscere chiaramente le mire, i disegni, e le occulte manovre di colui, che, resosi superiore ad ogni dritto, ha oltraggiato l'amicizia e la buona fede, e la lealtà dei Re e delle Nazioni. Si darà sempre una data di Messina per rapportar le notizie le più recenti, che arrivano per la via del mare, ed indi si sceglierà da' fogli stranieri ciò che sarà più importante, e più degno della cognizione del pubblico $»^{32}$.

A differenza degli altri giornali coevi dalla vita effimera, la Gazzetta resisteva a oltre sette anni di pubblicazioni, con uscite puntuali il mercoledì e il sabato, generalmente «un'ora prima di mezzogiorno», come si legge nell'avviso $A i$ Lettori, nel fascicolo numero 1, del 1813.

La redazione del giornale era affidata a illustri messinesi e, anche se non comparivano mai le firme, si sa dalle cronache del tempo che tra questi vi erano senza dubbio il padre Benedetto Chiavetta e il nobile Ottavio Saccano Nicolaci.

Seppure il giornale non apparisse come un vero e proprio organo di governo, la scelta della tipografia era sostanzialmente indicativa dell'uso che se ne voleva fare.

Giovanni del Nobolo, infatti, da sempre aveva stampato fogli a sostegno della monarchia borbonica e della presenza inglese nell'Isola definendosi sin dal 1806 "impressore britannico". L'uso di caratteri inglesi e la circostanza che

30. G. Cilestri dei Marchesi di Croce S., Elogio di I. Paternò Castello di Biscari. Catania, 1787.

31. Cfr. De Salvo, "Opinione pubblica, libertà di stampa e circolazione delle idee".

32. Cfr. Gazzetta Britannica, n. 1, 2 marzo 1808, Messina. Il testo completo del programma è ripreso da Arenaprimo, La stampa periodica, p. 47. 
qualche fascicolo, nel corso della vita del periodico, fosse stampato dalla Stamperia dell'Armata Britannica, non lascia spazio a dubbi.

La tipografia di del Nobolo era presumibilmente anche luogo d'incontro per quanti, impegnati politicamente, si trovavano a vivere con entusiasmo quegli anni caratterizzati dall'ideologia liberale inglese. Non era un caso, infatti, che presso quella bottega si potessero acquistare, durante gli anni dell'avventura costituzionale, i giornali provenienti da Malta, la Cronica di Sicilia e il Giornale Costituzionale di Palermo.

In quel contesto di affermazione delle idee costituzionali, non meraviglia che il del Nobolo si consacrasse definitivamente come tipografo "costituzionale" anche grazie alla pubblicazione in italiano della Costituzione politica della Monarchia spagnola. È noto, infatti, come il dibattito costituente spagnolo destasse particolare attenzione nell'attiva borghesia messinese che lo seguiva proprio attraverso le cronache riportate sulla Gazzetta Britannica.

\section{Brevi note conclusive}

Thomas Wright Vaughan, nell'introduzione all'edizione inglese del Giornale di viaggio di Paolo Balsamo, sosteneva che in Sicilia nel 1810,

non si pubblicano giornali ad eccezione della Gazzetta di Palermo, che ha solo estratti da giornali stranieri e non riporta alcuna notizia interna tranne gli arrivi e le partenze della famiglia reale; o della Gazzetta Britannica, che è stata da poco fondata a Messina, precisamente da quando ci sono gli Inglesi, e che naturalmente fornisce soltanto dettagli militari di interesse ben limitato, modellati sulle speranze e aspirazioni del suo redattore, che sono sinceramente patriottiche ${ }^{33}$.

In realtà mi sembra, alla luce di quanto detto, che il risalente giudizio storiografico, che voleva la pubblicistica siciliana del primo Ottocento scarsa nella qualità e poco qualificata rispetto a quella settentrionale, debba essere rivisto.

Notevole importanza, in tale prospettiva, rivestiva l'esperienza costituzionale del 1812, momento cruciale per «l'aprirsi della nostra coscienza politica verso le nuove aspirazioni di libertà ${ }^{34}$.

33. Si veda D'Angelo, M., "La "Gazzetta Britannica" di Messina e la Costituzione siciliana del 1812". Romano, A. (ed.), Il modello costituzionale inglese e la sua recezione nell'area mediterranea tra la fine del settecento e la prima metà dell'ottocento, Atti delle giornate di studio in memoria di Francisco Tomás y Valiente. Milano, 1998, p. 873. D'Angelo, M., "La Gazzetta Britannica, Saggio introduttivo". Molonia, G. (ed.), La Stampa periodica a Messina (1803-1863). Messina, 2004, pp. 7-42.

34. Mirabella, T., "Il giornalismo siciliano dell'Otto Novecento". Romeo, R., Storia della Sicilia IX, Napoli, 1978, pp. 299-355, in particolare p. 300. 
Di specifico rilievo era proprio il decreto costituzionale con il quale si sanciva la soppressione della censura preventiva e si proclamava la libertà di stampa:

«Ognuno potrà stampare, e pubblicare le sue idee senza il bisogno di licenza, e senza obbligo di sottoporle ad una precedente revisione, e ciò dal momento, che S. R. M. avrà sanzionato il presente Capitolo» ${ }^{35}$.

Un diritto importante per la formazione di un'opinione pubblica finalmente consapevole e in grado di far sentire la propria voce a livello politico.

Nell'ottica dell'affermazione e difesa dei diritti naturali, I'opinione pubblica diventava, dunque, testimone privilegiato di quel clima particolarmente dinamico.

Nel giro di pochi anni, erano edite, soprattutto a Palermo e Messina, una decina di testate che vivacizzavano, dalle loro pagine, il dibattito tra le varie correnti politiche (dai costituzionalisti ai reazionari intransigenti e al nascente partito democratico).

L'inesperienza dei redattori dei fogli, tuttavia, spesso non li rendeva in grado di adottare un linguaggio moderato e imparziale, lasciandoli cadere in invettive troppo appassionate.

Come aveva modo di evidenziare l'Evola, «la libertà di stampa è una conquista delle rivoluzioni, e non è facile stabilire se, concessa a popoli non educati ad esercitarla, abbia più giovato che nociuto».

In un momento di contrastanti vedute politiche, come si riveleranno i mesi successivi alla proclamazione della Costituzione, la libertà di stampa consentiva, infatti, il nascere di fogli polemici e di canzoni, di satire, di poesie anche contro gli uomini del potere.

La difficoltà di individuare l'estensione e i limiti di tale libertà, che seppure dichiarata costituzionalmente non trovava una tutela efficace nella sua applicazione, a causa della mancanza di un diritto certo e codificato ${ }^{36}$, è testimoniata anche dal diverso iter procedurale applicato ai numerosi processi per reati di stampa e "libello famoso", che spesso si trasformavano in veri e propri processi politici ${ }^{37}$.

Cercando di tirare le fila, quindi, mi domando se, in effetti, la tanto decantata libertà di stampa abbia avuto realmente la sua applicazione in Sicilia o se anche in questo caso si sia trattato solo di mera propaganda politica.

35. Costituzione del Regno di Sicilia. Palermo, 1813. Romano, A. (ed.), Ristampa anastatica e con Introduzione. Messina, 1996, p. 81.

36. Vedi Novarese, D., Costituzione e codificazione nella Sicilia dell'ottocento. II "progetto di codice penale" del 1813. Milano, 2000.

37. Sul punto, De Salvo, P., " "Sarà delitto il pubblicare scritti di qualunque sorta... che contengano libelli infamatori»: Un processo per violazione della libertà di stampa nella Sicilia costituzionale (1810-1815)". Acta Histrae, 19 (2011), 3, pp. 505-522. 
È vero che il numero delle pubblicazioni a stampa, delle gazzette e dei periodici, in quegli anni era notevolmente aumentato. Tuttavia, se si tralasciano i numerosi giornali ufficiali e i periodici culturali, poche erano le voci che uscivano dal coro come, ad esempio, il Giornale Patriottico fondato dal Barone Aceto a Palermo ${ }^{38}$. Tale valutazione mi sembra calzi perfettamente a proposito della stampa messinese. Infatti, la parzialità dell'informazione prodotta sulle pagine della Gazzetta Britannica, non era di molto inferiore a quella dei giornali filo-francesi della Penisola, anche se supportata e, a ciò bisogna dare rilievo, dall'esaltazione degli ideali d'indipendenza e dei principi liberali di marca anglosassone.

Sembra, dunque, potersi affermare che la libertà di stampa trovasse anche in quel frangente un baluardo invalicabile nella "ragion di stato" ovvero nella necessità di sostenere il primato politico della monarchia britannica nel Mediterraneo ${ }^{39}$.

La fine dell'età napoleonica, la conseguente partenza delle truppe inglesi dalla città dello Stretto e la creazione del Regno delle Due Sicilie portavano il giornalismo siciliano a segnare il passo.

Conseguenza di quelle vicende erano la vigilanza più rigorosa e la maggiore severità delle pene per i dissidenti politici. Si promettevano premi ai delatori mentre le perquisizioni diventavano sempre più arbitrarie; i giornali, per qualche tempo, non si occuparono più di politica ma pubblicavano per lo più articoli letterari e scientifici.

Bisognerà attendere la fiammata rivoluzionaria del 1820-1821 per rivedere, anche a Messina, fugaci lampi di libertà di stampa ${ }^{40}$.

38. Come ha avuto modo di sottolineare Francesco Brancato, non esiste su Giovanni Aceto uno studio complessivo e organico. Su questo importante personaggio siciliano si trovano soltanto dei cenni, in tutti gli scritti, editi ed inediti, riguardanti gli avvenimenti dell'isola dal 1812 al 1820. Cfr. Brancato, F., "Aceto Cattani Giovanni". Dizionario Biografico degli Italiani, 1, Roma, 1960. Si veda, inoltre, Aceto, G., "Il giornale patriottico" (1814-1816) e "il giornale patriottico di Sicilia" (1820). Antologia, con Introduzione di Berti, G., Palermo, 1969, pp. 7-27.

39. Cfr. De Salvo, "Opinione pubblica, libertà di stampa e circolazione delle idee".

40. De Salvo, P., "Civil and political liberties", pp. 456-459. 